\title{
Near field scattering for samples under forced flow
}

Cite as: Rev. Sci. Instrum. 91, 075108 (2020); https://doi.org/10.1063/1.5138694

Submitted: 15 November 2019 . Accepted: 23 June 2020 . Published Online: 07 July 2020

Llorenç Cremonesi (D), Mirko Siano (D), Bruno Paroli (D), and Marco A. C. Potenza (D)

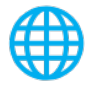

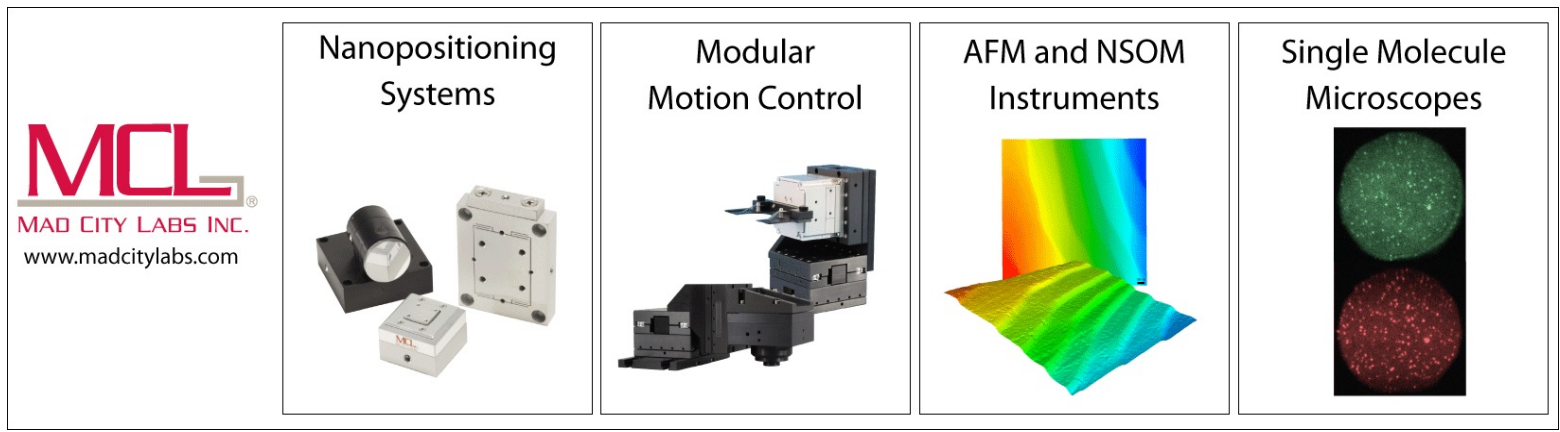




\title{
Near field scattering for samples under forced flow
}

\author{
Cite as: Rev. Sci. Instrum. 91, 075108 (2020); doi: 10.1063/1.5138694 \\ Submitted: 15 November 2019 - Accepted: 23 June 2020 • \\ Published Online: 7 July 2020
}

Llorenç Cremonesi, ${ }^{1, a)}$ (D) Mirko Siano,' (D) Bruno Paroli, '(D) and Marco A. C. Potenza ${ }^{1,2}$ (D)

\author{
AFFILIATIONS \\ ${ }^{1}$ Department of Physics, University of Milan, I-20133 Milan, Italy \\ ${ }^{2}$ CIMalNa, University of Milan, I-20133 Milan, Italy
}

a) Author to whom correspondence should be addressed: \|lorenç.cremonesi@unimi.it

\begin{abstract}
We describe a light scattering technique for characterizing colloidal samples under constant flow. It exploits the properties of speckles in the deep Fresnel region - the so-called near field speckles-providing absolute scattering measurements of the static form factor of the sample, as described extensively by Mazzoni et al. [Rev. Sci. Instrum. 84, 043704 (2013)] for static samples. We exploit a strongly astigmatic beam for illuminating the scattering volume with a light sheet a few microns thick. This largely improves the sensitivity of the method to small signals. Moreover, by flowing the sample in the direction perpendicular to the light sheet, the transit times are reduced to a minimum, allowing for fast measurements. We tested the instrument with suspensions of calibrated colloidal polystyrene spheres with a size comparable to the light wavelength. In particular, we recovered the static form factors of suspensions of spherical particles and the phase lag of the zero-angle scattering amplitude, which both compare well to Mie theory predictions. We then applied the method to colloidal fractal aggregates of sub-wavelength particles and measured their fractal dimension. The instrument is designed to be operational in continuous flow analysis systems.
\end{abstract}

Published under license by AIP Publishing. https://doi.org/10.1063/1.5138694

\section{INTRODUCTION}

In the last few years, considerable interest has been devoted to the development and application of optical methods to the study of nano- and microscopic particles suspended in fluids (Bigler et al., 2011; Potenza et al., 2012; Palberg, 2014; Ferri et al., 2015; Shimizu et al., 2016; and Garzonio et al., 2018). The main advantage of optical methods is to be non-invasive, thus allowing to monitor samples undergoing an extended class of phenomena. Among the optical methods, laser speckles and their properties have played a role since the advent of laser light, such as the ubiquitous dynamic light scattering. Giglio et al. (2000) designed a class of methods based on the formation of speckle fields just downstream of a random sample illuminated by spatially coherent laser light. These have been called near field speckles, since they are formed in the deep Fresnel region of the sample. At variance with the traditional far field speckles, their features depend on the scatterers: information about the two-point field-field correlation function of the field emitted by the sample can be grasped through a statistical analysis of the intensity distribution (Giglio et al., 2000). In the most commonly used realization of the method, speckles are formed by the (stochastic) interference between the faint light emerging from a random distribution of scattering centers and the intense, almost undisturbed transmitted beam (Brogioli et al., 2002; Ferri et al., 2004; and Mazzoni et al., 2013). The method thus relies on a robust self-reference interferometric optical scheme, where the intensity distribution is proportional to the scattered field amplitude of the particles in the sample. The power spectrum of the spatial intensity distribution of the speckles gives the power spectrum of the intensity as a function of the scattering angle, hence the name of the method, Near Field Scattering (NFS). This gives information about the two-point, density-density correlation function of the sample or the static form factor and allows us to perform absolute scattering measurements, as described extensively in Mazzoni et al. (2013). NFS has been extended to other wavelength ranges (Cerbino et al., 2008) with different applications such as the measurement of the spatio-temporal coherence properties of wavefronts (Alaimo et al., 2009; 2014; and Siano et al., 2015).

Among the advantages of near field scattering with respect to other scattering methods, we enumerate its stability and simplicity of 
the optical layout. Precise alignment of the optical components is not a critical requirement. The speckle size is constant with the distance along the optical axis so that the positioning of the collection plane is not very demanding. Moreover, a wide range of scattering angles can be recorded simultaneously (essentially limited by the collection optics), while stray light affecting measurements can be rigorously subtracted during data processing. Dedicated data processing further reduces the need for optical stability over time intervals of the order of the time lapse between two acquisitions (Mazzoni et al., 2013).

One of the main drawbacks of the NFS methods is the need to match a trade-off between two temporal conditions: (i) the exposure time must be short with respect to any change in the speckle pattern in order to prevent blurring and (ii) the time lag between two subsequent acquisitions must be longer than the time needed to refresh the entire speckle pattern, that is, to change the positions of the scattering centers enough to make the speckle patterns completely uncorrelated (Ferri et al., 2004). In principle, if a singleshot measurement is feasible, condition (ii) could be relaxed. This is not, however, the usual case-especially with visible light-for two concurrent reasons: multiple speckle patterns are needed to effectively subtract stray light and accumulating enough statistics usually requires collecting a large number of speckle patterns, particularly at the lowest scattering angles (Ferri et al., 2004).

Although, in some particular cases, this limitation on the exposure time and acquisition rate is not a concern (Siano et al., 2015), care is needed to guarantee an adequate trade-off. Depending on the kind of motion of the scattering centers, speckles can (a) translate following the sample flow in a direction transversal to the optical axis (Alaimo et al., 2006), (b) "boil" due to Brownian motions (Magatti et al., 2008) or motions along the optical axis, and (c) change due to Brownian rotations, which are a concern in the case of non-spherical objects (Potenza et al., 2014; Mazzoni et al., 2013). Any combination of these effects causes a change in the speckle pattern that must be either avoided or taken care of during data analysis. Dealing with Brownian motions can be particularly critical, since the minimum time scale is imposed by the size of the single scattering center or its internal structures that are in most cases unknown during the measurement. Moreover, in the case of limited spatial coherence as it is with non-optical radiation (Bonn et al., 2009), speckle stability can depend on more complex features arising from the combination of the dynamics of the sample and the source coherence (Siano et al., 2015). In order to measure the static form factor, it could be convenient to flow the sample through the scattering volume, as it is common practice in traditional low-angle light scattering (LALS) measurements. This guarantees a fast refresh of the sample, reducing the time required for reliable measurements. In a traditional NFS configuration, this would introduce substantial motion blurring, canceling out part of or possibly all of the speckle field. We stress that by implementing the NFS optical scheme with a typical low power laser $(5 \mathrm{~mW})$, preventing motion blur by simply reducing the exposure time is not a viable option because of the limited sensitivity of common imaging sensors.

Here, we report an optical layout capable of effectively overcoming this limitation and appreciably improving the sensitivity, still maintaining the basic approach and the advantages of the NFS method. In order to prevent the signal from motion blurring, the cell is transilluminated by an extremely astigmatic laser beam, focused into the flow cell with the focal line orthogonal to the sample flow (here assumed to be along the vertical direction). We largely optimize the fast renewal of the scattering centers by strongly reducing the extension of the illuminated region along the flow direction. The vertical extension of the focal line imposes the minimum time required for all the scattering centers to move outside the illuminated region and consequently the minimum time-lag between two subsequent acquisitions.

In terms of the system sensitivity, this approach introduces an important advantage. In the traditional NFS with plane wave illumination, the transmitted beam intensity and amplitude are constant. The beam heterodynes the scattered fields emitted as spherical waves, with their amplitudes decreasing considerably as the distance of the observation plane from the scattering region increases. In the present astigmatic case, the imbalance between the transmitted and the power of the scattered field is partially compensated: the beam expansion in the vertical direction reduces the intensity and the corresponding field amplitude. Scattered fields are emitted by particles illuminated by the intense focal line, and the propagated spherical wavefronts are heterodyned by the transmitted field that is much fainter compared to the plane wave case. As a consequence, the contrast of the interference patterns is appreciably enhanced with respect to the traditional NFS configuration, and the system sensitivity is appreciably increased, making it much easier to measure very diluted samples, for example.

\section{OPTICAL LAYOUT AND FUNDAMENTALS OF THE METHOD}

A schematic of the optical layout is shown in Fig. 1. A He$\mathrm{Ne}$, collimated, Gaussian beam (632.8 nm wavelength, $5 \mathrm{~mW}$ power, and $0.5 \mathrm{~mm}$ beam waist) is spatially filtered, expanded, and collimated through an achromatic doublet, $50 \mathrm{~mm}$ in focal length. The beam, $\sim 8 \mathrm{~mm}$ in diameter, is then focused by a cylindrical lens (focal length $f=80 \mathrm{~mm}$ ), with the optical power in the vertical direction. It shapes the beam into a focal line with its major and minor semi-axes of $4 \mathrm{~mm}$ (horizontal, $\mathrm{H}$ ) and $\sim 10 \mu \mathrm{m}$ (vertical, $\mathrm{V}$ ), respectively. The effective horizontal size is limited by the width of the flow cell. The vertical size of the light sheet adopted here is chosen to be larger than the particles in the samples under consideration. It should be noted that if a particle is larger than the vertical size of the light sheet, the S(q) might not, in general, be measured correctly: one should ensure that the number of such events is negligible. The focal line is precisely placed at the center of a plane parallel quartz flow-cell (Hellma 170.700 QS, $200 \mu \mathrm{m}$ optical path) flown through by the sample to be examined. This is the only alignment that is critical to the proper functioning of the technique. It is also worth noting that the sample (cell) thickness is much smaller than the Rayleigh range of the focal line so that the scattering centers are all illuminated by plane waves with a negligible Gouy phase shift. The emerging beam and the scattered wavefronts are collected $10 \mathrm{~mm}$ downstream the sample in the observation plane. This plane is placed much further than the Rayleigh range of the focal line, thus guaranteeing the far field conditions in the $\mathrm{V}$ direction while maintaining the deep Fresnel conditions for the $\mathrm{H}$ direction. A collection optics composed by an afocal, long distance microscope objective (Edmund Optics 59 878, $20 \times$ ) and a field lens doublet forms a $15 \times$ magnified image of the 


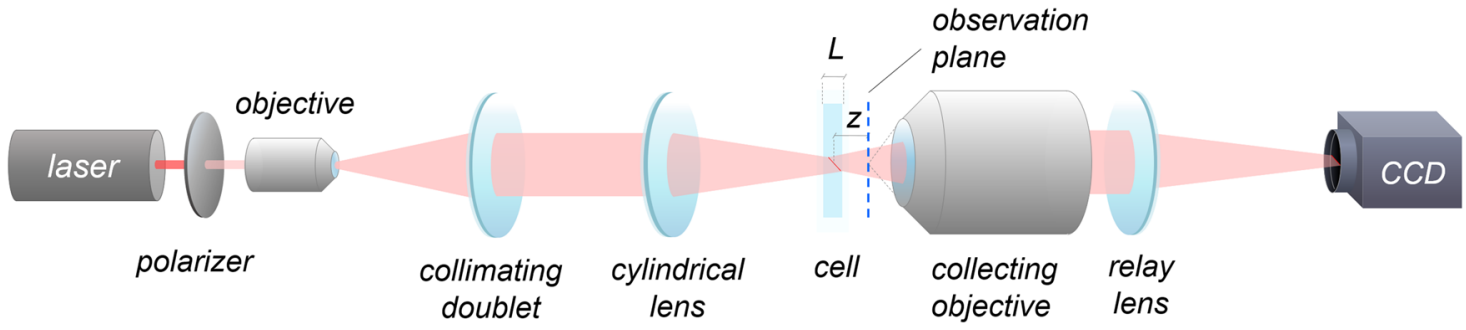

FIG. 1. Schematic of the optical layout. A He-Ne laser $(632.8 \mathrm{~nm})$ beam is spatially filtered and expanded through a $20 \times$ objective and a collimating doublet, $50 \mathrm{~mm}$ focal length. A cylindrical lens focuses the beam in the vertical direction at the center of a flow cell. The emerging light is collected at a distance of $z=10 \mathrm{~mm}$ by a microscope objective, and a relay lens magnifies the image onto the CCD sensor.

observation plane onto the surface of a CCD sensor. Either a 2D pixelated sensor or a linear, 1D pixel array has been adopted to the aim, as described below.

For the sake of clarity, let us refer to the observation plane, the sensor being just collecting light intensity magnified through the microscope. We will also restrict our considerations within a limited extension in the $\mathrm{V}$ direction of the observation plane. This is particularly useful, especially from an experimental point of view, since a sensor with the limited $\mathrm{V}$ extension offers substantial advantages in terms of the acquisition speed: the number of pixels to be red is much less than in the traditional NFS. Note that the focal line illuminates only particles within a very thin region in the $\mathrm{V}$ direction, elongated in the $\mathrm{H}$ direction orthogonally to the optical axis.

Let us now briefly describe the phenomena in the focal region and the observation plane from a more formal point of view. We consider an astigmatic laser beam of wavelength $\lambda$ focused in the $y$ direction (V direction) by a cylindrical lens of focal length $f$. The vertical extension of the beam at the focal line $(z=0)$ and at the observation plane at a distance $z$ are

$$
w_{0}=\frac{\lambda f}{\pi w_{L}}, \quad w(z)=w_{0} \sqrt{1+\left(\frac{z}{z_{R}}\right)^{2}},
$$

respectively, where $w_{L}$ is the width of the collimated laser beam impinging on the cylindrical lens, while the Rayleigh range, $z_{R}$, in a medium with refractive index $n$ is defined as usual,

$$
z_{R}=n \frac{\pi w_{0}^{2}}{\lambda} .
$$

By using the properties of Gaussian beams, the field amplitude impinging onto the particles within the focal line $\left(|y|<w_{0}\right.$ and $|z|$ $\left.<z_{R} ; k=2 \pi / \lambda\right)$ is

$$
E_{0} \simeq \sqrt{\frac{w_{L}}{w_{0}}} e^{i\left(k z-\frac{1}{2} \arctan \left(z / z_{R}\right)\right)} .
$$

On the other hand, on the observation plane, we can set $y=0$ and assume $z \gg z_{R}$, hence $\arctan \left(z / z_{R}\right) \simeq \frac{\pi}{2}$. We then obtain the reference field amplitude,

$$
E_{R}=\sqrt{\frac{w_{L}}{w(z)}} e^{i\left(k z-\frac{\pi}{4}\right)},
$$

and note that the astigmatic beam determines a phase shift of $\frac{\pi}{4}$, at variance with the value $\frac{\pi}{2}$ of a stigmatic one. Without entering into more details, we just note that, on the observation plane, the superposition of the reference field (4) and the field scattered by objects illuminated by the field (3) will generate an intensity distribution. Dividing by the incident intensity, $\mathrm{I}_{0}$, this assumes the following form:

$$
\begin{aligned}
I(x) & =\mid 1+\sqrt{\frac{w(z)}{w_{0}}} \sum \text { spherical waves }\left.\right|^{2} \\
& \simeq 1+2 \sqrt{\frac{w(z)}{w_{0}}} \sum \text { spherical waves. }
\end{aligned}
$$

The prefactor $\sqrt{w(z) / w_{0}}$ of the interference term shows the enhancement of the contrast mentioned above. By introducing the formal expressions for the spherical waves emerging from $N$ (identical) scattering centers within the focal line, with some mathematics, one obtains the power spectrum $P(q)$ of the interference term in the intensity distribution [Eq. (5)]. This provides the static form factor multiplied by the enhancing term and the Talbot transfer function, $T(q)$, typical of deep Fresnel speckles (Mazzoni et al., 2013),

$$
P(q)=N \frac{\lambda}{z} \frac{|S(q)|^{2}}{k^{2}} \cdot \frac{w(z)}{w_{0}} \cdot T(q),
$$

where $S(q)$ represents the adimensional amplitude of the field scattered at a wavevector $q=2 k \sin (\theta / 2)$ corresponding to a scattering angle $\theta$, as defined in Van de Hulst (1957). The transfer function $T(q)$ arises from the interference of multiple, spherical waves arising from the scattering volume. A complete description of $T(q)$ is given in Eq. (7); further details can be found in Potenza et al. (2010) and Mazzoni et al. (2013). We note that since $w(z) \sim z$ in the far field, $P(q)$ is independent of the distance from the sample, $z$. This feature makes absolute scattering measurements possible even in the present case of astigmatic illumination. Therefore, the setup introduced here is able to reproduce the performances of the traditional NFS method, with the added advantage of an enhanced sensitivity that is, within some limits, even tunable.

Following the two approaches described in Ferri et al. (2004), Mazzoni et al. (2013), and Potenza et al. (2010), we exploit the selfreference interferometric scheme to extract independent information about the static form factor from the spatial power spectrum (6) and the phase lag $\varphi=\operatorname{ArgS}(0)$ of the scattered waves from the Talbot oscillations, where $S(0)$ is the scattering amplitude of the particle (Van de Hulst, 1957). The optical layout described here gives access 
to the power spectrum up to $q \sim 4 \mu \mathrm{m}^{-1}$. Two wavevector ranges shall be considered: for $q>0.6 \mu \mathrm{m}^{-1}$, the power spectrum gives the static form factor of the scattering centers (Ferri et al., 2004; Mazzoni et al., 2013). In the case of fractal aggregates, the asymptotic behavior of the static form factor gives the independent measurement of the fractal dimension (Weitz and Lin, 1986; Ferri et al., 2004; and Mazzoni et al., 2013). On the other hand, the low- $q$ region $(q<0.6$ $\left.\mu \mathrm{m}^{-1}\right)$ is characterized by Talbot oscillations, delivering information about the phase lag of the scattered waves. Following Potenza et al. (2010) and Mazzoni et al. (2013), the experimental data in the low- $q$ region are fitted by the following function:

$$
T(q)=1+\cos \left(\frac{q^{2}}{k} \tilde{z}-2 \varphi\right) \cdot \sin c\left(\frac{q^{2}}{2} \frac{L}{n k}+\frac{\pi}{8} \frac{L}{z_{R}}\right),
$$

where $\tilde{z} \simeq z+\frac{L}{2 n}$ is the effective distance between the focal line and the observation plane, which takes into account the optical path between the source and the observation plane, $L$ is the cell thickness, and $n$ is the refractive index of water. The term including $z_{R}$, defined as in Eq. (2), is due to the focused incident field instead of a simple plane wave. The phase-lag introduced by the particles, $\varphi$, is the only free parameter to be fitted to experimental data; it reaches values close to $-\frac{\pi}{2}$ in the Rayleigh regime up to values close to zero for large particles falling in the diffraction regime. However, and most interestingly, the phase-lag depends on the optical thickness of the scatterer (Van de Hulst, 1957).

\section{CALIBRATION AND EXPERIMENTAL RESULTS}

The performances of the system have been evaluated by means of many tests with diluted colloidal suspensions under different flow conditions.

We report here the results obtained with diluted colloidal samples constituted by monodisperse suspensions of polystyrene spheres $0.6 \mu \mathrm{m}, 2 \mu \mathrm{m}$, and $2.9 \mu \mathrm{m}$ in diameter, almost monodisperse $(\sigma /\langle d\rangle=3 \%, 4 \%$, and $5 \%$, respectively). Data are compared to the corresponding Mie curves. Two acquisition approaches have been adopted: (1) a digital CCD camera recording a region of interest (ROI) $1048 \times 16$ pixels $^{2}$, small enough to be transferred and stored in short times allowing a fast acquisition of independent frames (100 frames/s); (2) a digital mono-dimensional CCD array with 2000 pixels, transferring up to $18 \mathrm{k}$ frames/s to accelerate the fluid flow and acquisition rate further. The former, two-dimensional sensor is much more convenient in terms of the alignment procedure and signal to noise ratio. Averaging the pixel values along the vertical direction reduces the signal noise (mainly caused by shot noise). In Fig. 2, an example of the intensity distribution within the ROI is shown. The latter sensor suffers from the lack of vertical information on the observation plane, which is a strong limitation in the alignment procedure, although allowing for a significant reduction in the measurement time. The results reported here have been obtained with the former approach.

The constant flow is obtained by draining the sample from a container at normal pressure, as in Alaimo et al. (2006). A $2 \mathrm{~mm}$ internal diameter tube delivers the liquid to the quartz flow-cell. A flow of $\sim 1 \mathrm{ml} / \mathrm{min}$ provides a speed of $20 \sim \mathrm{mm} / \mathrm{s}$ at the center of the cell (i.e., at the maximum speed of the Poiseuille flow). Consistently with the width of the focal line, the time required to renew the sample in the illuminated region shortens down to $\sim 1 \mathrm{~ms}$. When operating the one-dimensional CCD array, the speed was increased by more than an order of magnitude, allowing the acquisition rate to be increased accordingly. Tuning the fluid speed into the cell allows imposing different shear conditions to the sample. When measuring colloidal aggregates, we exploited this possibility to find proper conditions under which their structure factor was not appreciably affected by shear. More generally, this technique may be used to check the effects of shear stress on flowing samples populated by fragile particles such as aggregate structures.

As described in Ferri et al. (2004) and Mazzoni et al. (2013), calibration is needed in order to characterize, and compensate for, the modulation transfer function (MTF) of the whole optical system. This clearly affects the amplitude of the interference patterns at different $q$ values and therefore the measured power spectrum and the corresponding static form factor, hereinafter indicated with $S_{s}(q)$.

The calibration procedure is straightforward. We first acquired an extensive dataset with a flow of pure, filtered water as a baseline. The average power spectrum has then subtracted to all the spectra retrieved afterward. We then measured calibrated spherical particles with an almost flat power spectrum. The particle size was chosen in order to avoid unnecessary features in the expected spectrum while ensuring a good signal to noise ratio. In Fig. 3, we report the power spectrum of the NFS speckles formed by polystyrene spheres $600 \mathrm{~nm}$ in diameter (Thermo Fisher Scientific). Experimental (uncalibrated) data are reported as green dots, compared with the Mie curve [black solid line in Fig. 3(a)]. The plots are the normalized average of the power spectra obtained from $10^{4}$ independent images (frames), each averaged over the 10 pixel vertical extension of the ROI.

For $q>0.6 \mu \mathrm{m}^{-1}$, data have been adapted to the expected Mie curve by multiplying by a smooth function [solid gray line in Fig. 3(a)]. This gives the MTF needed to calibrate the power spectra, whereby we obtain the calibrated experimental data (red squares) with the corresponding error bars (the pink area).

In Fig. 3(b), details of the oscillations in the low- $q$ region $\left(q<0.6 \mu \mathrm{m}^{-1}\right)$ are given (red dots connected by the red solid line). Oscillations ascribable to the Talbot transfer function are well visible up to approximately $q \sim 0.6 \mu \mathrm{m}^{-1}$ : the phase lag of the particles in the sample can be retrieved by fitting Eq. (7) to data (green solid

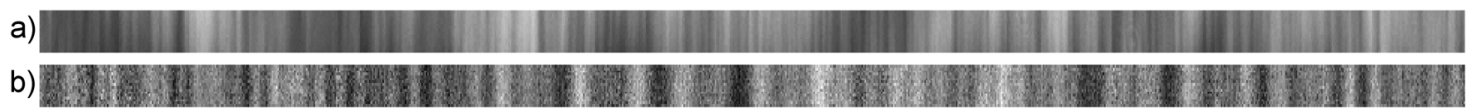

FIG. 2. (a) Example of an image captured by the camera $16 \times 1024$ pixels ${ }^{2}$. The width of the image is $6.8 \mathrm{~mm}$. Note that the intensity modulation is almost uniform vertically. (b) The same image after removing the baseline, revealing the speckle field. The images are reported enlarged in the vertical direction for graphical clarity. 

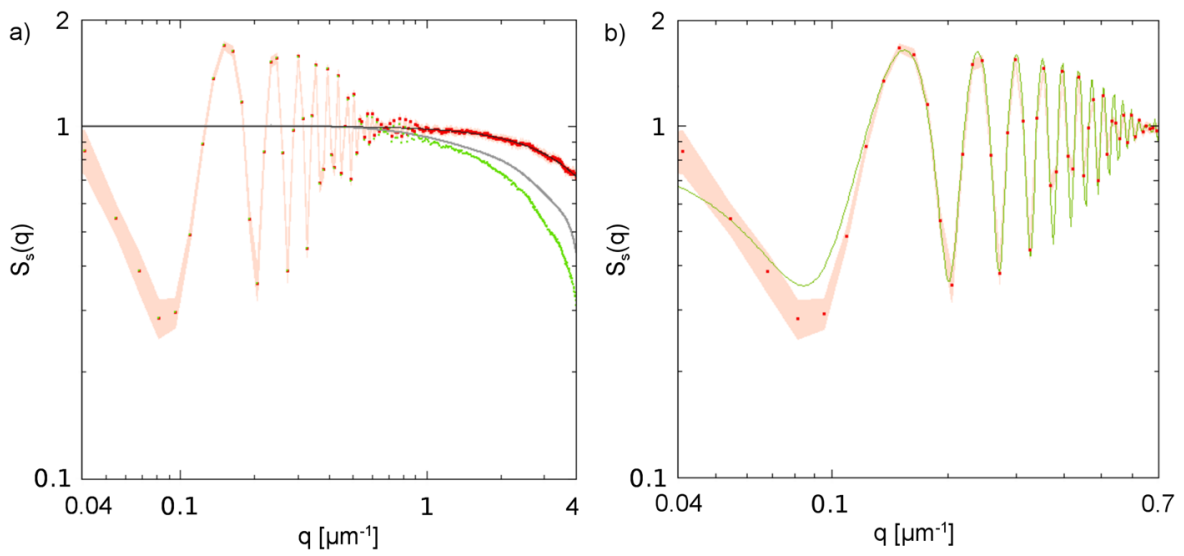

FIG. 3. (a) Average of 10 normalized spectra obtained with polystyrene particles $600 \mathrm{~nm}$ in diameter (red squares), compared to the corresponding Mie curve (black line). The pink area corresponds to the error bars. This measurement has been used for calibrating the instrument. For comparison, uncalibrated data are shown as green dots, whereas the MTF is reported as a solid gray line. (b) Details of the oscillations in the low q region. Talbot-like oscillations are well visible up to $\mathrm{q} \sim 0.6 \mu \mathrm{m}^{-1}$ The phase lag can be retrieved by fitting Eq. (7) to data (green solid line) and gives $\varphi \simeq-1 \mathrm{rad}$, in good agreement with the expected value.

line). This yields $\varphi=-1.00 \pm 0.03 \mathrm{rad}$, in good agreement with the expected value $\varphi \simeq-1.05 \mathrm{rad}$. We stress that this result does not depend on the calibration, since it involves the lower range of $q$ : it constitutes an independent result.

The calibration has been validated with polystyrene beads $500 \mathrm{~nm}$ in diameter, with equivalent results: compensating raw data with the MTF obtained above gave good agreement with Mie theory. Moreover, by fitting data with Eq. (7), we obtained $\varphi=-1.10 \pm 0.03$ rad, compared with $\varphi=-1.15 \mathrm{rad}$. Here, we report the results from larger particles, which exhibit some interesting distinctive traits in their spectra. In Figs. 4 and 5, experimental results are reported for colloidal samples $2.0 \mu \mathrm{m}$ and $2.9 \mu \mathrm{m}$ in diameter, respectively. The same data analysis procedure has been followed: the baseline has been removed, and the MTF has been compensated for with the calibration curve obtained above. Results are then compared to the Mie curves without any free parameter with which data are in good agreement. In this respect, we note the discrepancy close to the local minima at $q=3.3 \mu \mathrm{m}^{-1}$ and $q=1.8 \mu \mathrm{m}^{-1}$, respectively, may be ascribed to the small size polydispersity of the colloidal samples, causing a slight variability of the wavevector at which the intensity vanishes. Subsequent measurements all gave the same result. Fitting the Talbot oscillations to data gives $\varphi=0.27 \pm 0.05 \mathrm{rad}$ and $\varphi=0.33$ $\pm 0.05 \mathrm{rad}$, respectively, a mild overestimation of the phase lag of the particles. This small discrepancy with respect to theory may be ascribed to polydispersity as well.

Finally, we tested the instrument on samples containing fractal structures formed by the salt-induced aggregation process in a water suspension of polystyrene beads. Samples have been prepared as follows: Monodisperse polystyrene spheres of sub-wavelength diameter (100 nm monomers, Thermo Fisher Scientific, 3100A) were suspended in a beaker of pure water at room temperature. The number concentration was $\sim 5 \cdot 10^{10}$ particles per ml. Aggregation was triggered by adding a water solution of salt (magnesium chloride, $\mathrm{MgCl}_{2}$ ) to the suspension. Adapting the procedure used by Carpineti et al. (1990) to our samples, we employed a water solution of $\mathrm{MgCl}_{2}$ at a concentration $38 \mathrm{mM}$, which ensures aggregation in some minutes following the Diffusion Limited Aggregation (DLA) process [see Witter and Sander (1983)]: the aggregation characteristic time was of the order of $3 \mathrm{~s}-4 \mathrm{~s}$. At the very early stages of the process, the suspension was mixed by slowly spinning the beaker. The samples were ready to be measured after a few tens of minutes: small portions of the sample were gently picked up at different stages of the aggregation process and diluted with pure water 1 to $5-10$, in order to stop the aggregation process, and then
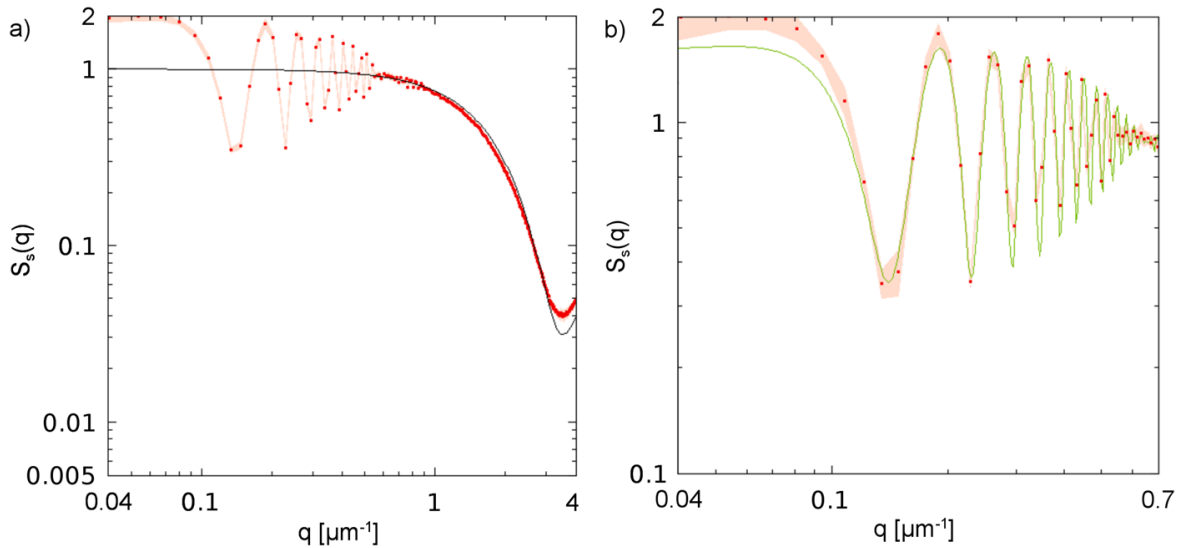

FIG. 4. (a) Average of 10 spectra obtained with polystyrene particles $2 \mu \mathrm{m}$ in diameter (red squares connected by the red solid line). The filled area corresponds to the experimental error bars. The data are in good agreement with the corresponding Mie curve (black line). (b) Enlargement of the oscillations in the low q region fitted by Eq. (7) (green solid line). 

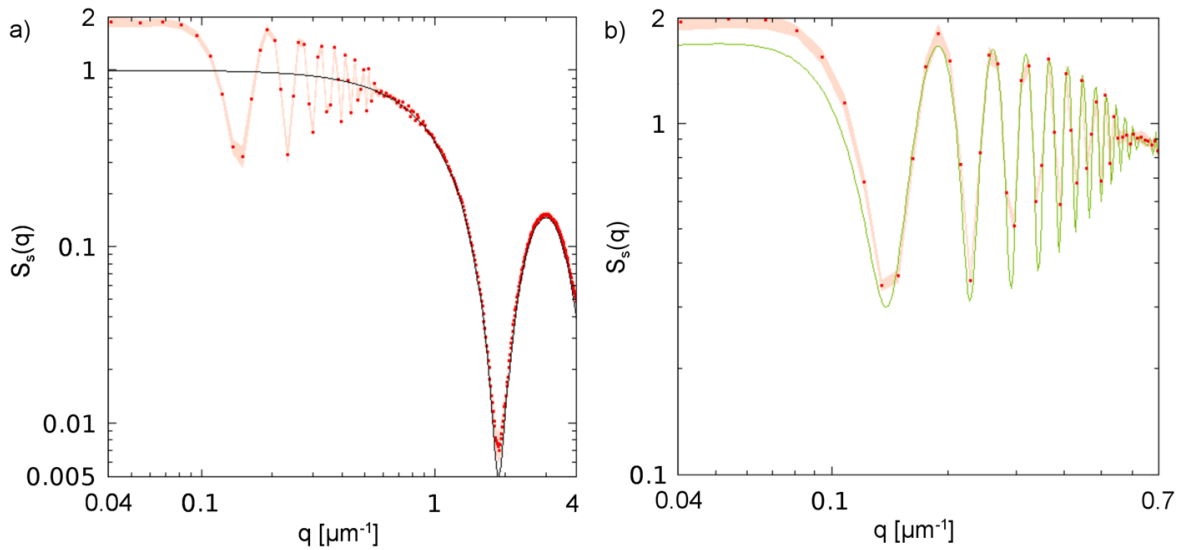

FIG. 5. Same as in Fig. 4 for spectra obtained with polystyrene particles 2.9 $\mu \mathrm{m}$ in diameter.
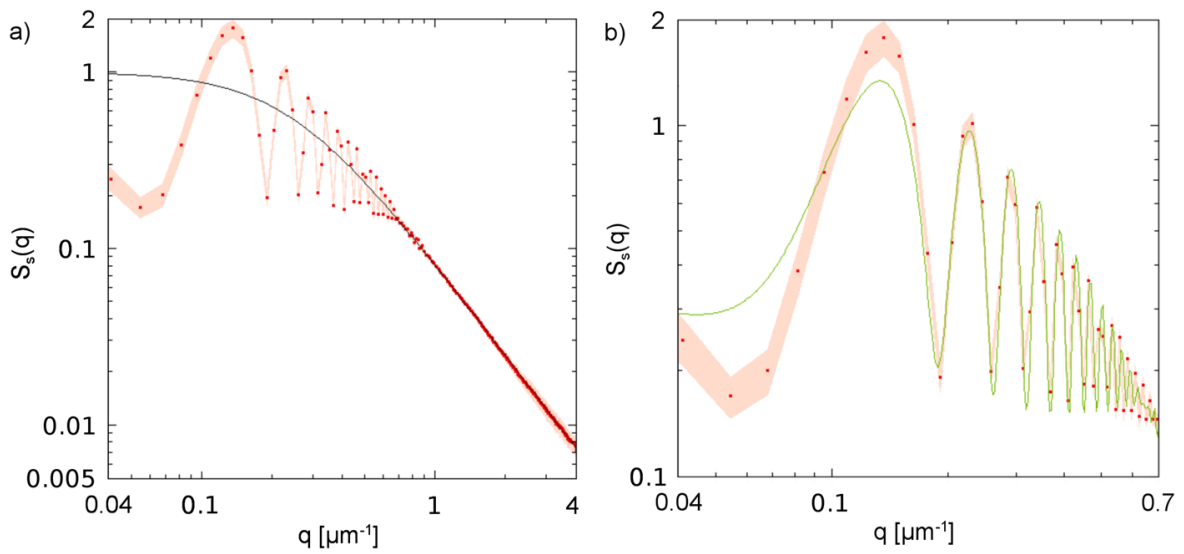

FIG. 6. (a) NFS data from colloida fractal aggregates formed by $100 \mathrm{~nm}$ polystyrene beads after $50 \mathrm{~min}$ at room temperature (red squares). The pink area corresponds to the experimental error bars. Fitting data with a FisherBurford curve (black solid line), the slope of the spectrum at high $q$ is compatible with a fractal dimension of $d_{f}=1.76$. (b) Talbot-like oscillations provide the phase lag by fitting data with Eq. (7) (green solid line) about $0.2 \pm 0.05 \mathrm{rad}$.

measured immediately. Samples have been flown at a flow rate of $\sim 0.7 \mathrm{ml} / \mathrm{min}$, which correspond to a speed of $\sim 20 \mathrm{~mm} / \mathrm{s}$ through the flow cell.

The results of the measurements on colloidal aggregates are shown in Fig. 6. Despite the size polydispersity, the oscillations at low $q$ are well visible in each measurement (Fig. 6). This suggests that the phase lag of the field amplitude is almost the same for all the aggregates in the population; otherwise, the oscillations would cancel out, as discussed in Potenza et al. (2010). As with the calibrated samples in Figs. 3-5, polydispersity affects the agreement of experimental data to the model in Eq. (7) at low q, reducing the accuracy of the estimate of the phase-lag. This is even enhanced here due to the size polydispersity of the aggregates. Measurements taken at several aggregation stages consistently give the same phase lag, $\varphi=0.16 \pm 0.03 \mathrm{rad}$. We stress that this value is appreciably larger than the phase-lag for $100 \mathrm{~nm}$ polystyrene monomers, i.e., $\varphi=0.02 \mathrm{rad}$, as expected for scatterers with an internal structure (Sorensen, 2001).

The structure factor of such particles is commonly approximated by a Fisher-Burford curve. As a matter of fact, many models are concurrently adopted in the literature (Sorensen, 2001). Since here we are only interested in the fractal dimension of the population, we analyze the power law trend of the spectra at high$q$, which is independent of the specific model. In our log-log plots, we just evaluate the slope: the high- $q$ asymptotic behavior yields a fractal dimension of $d_{f}=1.76$, which is compatible with the expected value for such systems (Weitz and Lin, 1986) and in agreement with independent measurements performed with small angle light scattering (Ferri et al., 1989). Furthermore, this is an indication that the imposed flow does not damage the structure of such particles appreciably. We repeated the procedure outlined above with aggregates of particles $70 \mathrm{~nm}$ in diameter with equivalent results. By our initial observations, flow rates higher than $\sim 10 \mathrm{ml} / \mathrm{min}$ yield spectra that lose the well-defined, straight trend in the log-log plots, which typify fractal aggregates. This suggests a possible disruption/compaction of the fractal aggregates under shear. Such a flow rate corresponds to a shear stress of the order of $10^{-1} \mathrm{~Pa}$.

\section{CONCLUSIONS}

In this work, we have described a non-invasive, robust optical technique based on the self-reference near field scattering method, adapted to work on colloidal samples under imposed flow of up to some $\mathrm{ml} \cdot \mathrm{min}^{-1}$ at a speed of tens of $\mathrm{mm} / \mathrm{s}$. The instrument is suitable 
to work in line with other optical or electronic instruments, hence in continuous flow analysis systems, for example. From the power spectrum of the randomly speckled intensity fluctuations, we are able to retrieve the absolute measurement of the static form factor over a wavevector range from $0.02 \mu \mathrm{m}^{-1}$ to $4 \mu \mathrm{m}^{-1}$ and the phase lag of the forward scattering amplitude. The method can be successfully used on polydisperse samples characterized by a complex structure as the fractal aggregates considered here. Thanks to the highly astigmatic beam, the scattering volume covers the whole cell width, maximizing the throughput of the system. Moreover, focusing in the direction parallel to the flow ensures an appreciable optimization of the contrast, ultimately enhancing the sensitivity. This optical layout is suitable for measurements of diluted suspensions characterized by low particle concentrations; the vertical size of the light sheet should be chosen according to the desired application. A tight focusing favors a high signal to noise ratio and extends downward the lower size limit for the particles detectable by the instrument. Similarly, the beam waist establishes an upper size limit, as large objects may surpass the vertical size of the light sheet. Finally, a possible application of this device may also include the study of the effects of shear on soft aggregate samples. From a more technical point of view, the number of optical components is kept to a minimum and the system does not require any precise alignment and no more than a sporadic check of the calibration.

\section{ACKNOWLEDGMENTS}

We acknowledge Mr. Daniele di Roberto for supporting early developments of the method described here.

\section{DATA AVAILABILITY}

The data that support the findings of this study are available from the corresponding author upon reasonable request.

\section{REFERENCES}

Alaimo, M. D., Magatti, D., Ferri, F., and Potenza, M. A. C., "Heterodyne speckle velocimetry," Appl. Phys. Lett. 88, 191101 (2006).

Alaimo, M. D., Potenza, M. A. C., Manfredda, M., Geloni, G., Szutcki, M., Narayanan, T., and Giglio, M., "Probing the transverse coherence of an undulator X-ray beam using Brownian particles,” Phys. Rev. Lett. 109, 194805 (2009).

Alaimo, M. D. et al., "Mapping the transverse coherence of the self amplified spontaneous emission of a free-electron laser with the heterodyne speckle method," Opt. Express 22, 030013 (2014).

Bigle, M., Svensson, A., Kettner, E., Vallelonga, P., Nielsen, M. E., and Steffensen, J. P., "Optimization of high-resolution continuous flow analysis for transient climate signals in ice cores," Environ. Sci. Technol. 45, 4483 (2011).

Bonn, D., Otwinowski, J., Sacanna, S., Guo, H., Wegdam, G., and Schall, P., “Direct observation of colloidal aggregation by critical casimir forces," Phys. Rev. Lett. 103, 15610 (2009).
Brogioli, D., Vailati, A., and Giglio, M., "Heterodyne near field scattering," Appl. Phys. Lett. 81, 4109 (2002).

Carpineti, M., Ferri, F., Giglio, M., Paganini, E., and Perini, U., "Salt-induced fast aggregation of polystyrene latex,” Phys. Rev. A 42, 7347 (1990).

Cerbino, R., Peverini, L., Potenza, M. A. C., Robert, A., Bösecke, P., and Giglio, M., "X-Ray-scattering information obtained from near-field speckle," Nat. Phys. 4, 238 (2008).

Ferri, F., Calegari, G. R., Molteni, M., Cardinali, B., Magatti, D., and Rocco, M., "Size and density of fibers in fibrin and other filamentous networks from turbidimetry: Beyond a revisited Carr-Hermans method, accounting for fractality and porosity," Macromolecules 48, 5423 (2015).

Ferri, F., Giglio, M., and Perini, U., "Inversion of light scattering data from fractals by the Chahine iterative algorithm," Appl. opt. 28, 3074 (1989).

Ferri, F., Magatti, D., Pescini, D., Potenza, M. A. C., and Giglio, M., "Heterodyne near-field scattering: A technique for complex fluids,” Phys. Rev. E 70, 041405 (2004).

Garzonio, R., Di Mauro, B., Cogliati, S., Rossini, M., Panigada, C., Delmonte, B., Maggi, V., and Colombo, R., "A novel hyperspectral system for high resolution imaging of ice cores: Application to light-absorbing impurities and ice structure,” Cold. Reg. Sci. Technol. 155, 47 (2018).

Giglio, M., Carpineti, M., and Vailati, A., "Space intensity correlations in the near field of the scattered light: A direct measurement of the density correlation function $g(r)$," Phys. Rev. Lett. 85, 1416 (2000).

Magatti, D., Alaimo, M. D., Potenza, M. A. C., and Ferri, F., "Dynamic near field scattering," Appl. Phys. Lett. 92, 241101 (2008).

Mazzoni, S., Potenza, M. A. C., Alaimo, M. D., Veen, S. J., Dielissen, M., Leussink, E., Dewandel, J.-L., Minster, O., Kufner, E., Wegdam, G., and Schall, P., "SODI-COLLOID: A combination of static and dynamic light scattering on board the International Space Station,” Rev. Sci. Instrum. 84, 043704 (2013).

Palberg, T., "Crystallization kinetics of colloidal model suspensions: Recent achievements and new perspectives," J. Phys.: Condens. Matter 26, 333101 (2014).

Potenza, M. A. C., Manca, A., Veen, S. J., Weber, B., Mazzoni, S., Schall, P., and Wegdam, G. H., "Dynamics of colloidal aggregation in microgravity by critical casimir forces," Europhys. Lett. 106, 68005 (2014).

Potenza, M. A. C., Sabareesh, K. P. V., Carpineti, M., Alaimo, M. D., and Giglio, M., "How to measure the optical thickness of scattering particles from the phase delay of scattered waves: Application to turbid samples," Phys. Rev. Lett. 105, 193901 (2010).

Potenza, M., Sanvito, T., Degiorgio, V., and Giglio, M., "Confocal depolarized dynamic light scattering,” Adv. Chem. Phys. 151, 61 (2012).

Shimizu, M., Saito, T., Nishiyama, Y., Iwamoto, S., Yano, H., Isogai, A., and Endo, T., "Fast and robust nanocellulose width estimation using turbidimetry," Macromol. Rapid Commun. 37, 1581 (2016).

Siano, M., Paroli, B., Chiadroni, E., Ferrario, M., and Potenza, M. A. C., "Measurement of power spectral density of broad-spectrum visible light with heterodyne near field scattering and its scalability to betatron radiation," Opt. Express 23, 32888 (2015).

Sorensen, C. M., "Light scattering by fractal aggregates: A review," Aerosol Sci. Technol. 35, 648 (2001).

Van de Hulst, H. C., Light Scattering by Small Particles (Dover, New York, 1957).

Witter, T. A. and Sander, L. M., "Diffusion-limited aggregation," Phys. Rev. B 27, 5686 (1983).

Weitz, D. A. and Lin, M. Y., "Dynamic scaling of cluster-mass distributions in kinetic colloid aggregation,” Phys. Rev. Lett. 57, 2037 (1986). 